\title{
Anatomic Reconstruction of Distal Radioulnar Ligaments With Tendon Graft for Treating Distal Radioulnar Joint Instability: Surgical Technique and Outcome
}

\author{
Meyer, Daniela ; Schweizer, Andreas ; Nagy, Ladislav
}

DOI: https://doi.org/10.1097/BTH.0000000000000163

Posted at the Zurich Open Repository and Archive, University of Zurich

ZORA URL: https://doi.org/10.5167/uzh-140553

Journal Article

Published Version

Originally published at:

Meyer, Daniela; Schweizer, Andreas; Nagy, Ladislav (2017). Anatomic Reconstruction of Distal Radioulnar Ligaments With Tendon Graft for Treating Distal Radioulnar Joint Instability: Surgical Technique and Outcome. Techniques in Hand Upper Extremity Surgery, 21(3):107-113.

DOI: https://doi.org/10.1097/BTH.0000000000000163 


\title{
Anatomic Reconstruction of Distal Radioulnar Ligaments With Tendon Graft for Treating Distal Radioulnar Joint Instability: Surgical Technique and Outcome
}

\author{
Daniela Meyer, MD, Andreas Schweizer, MD, and Ladislav Nagy, MD
}

\begin{abstract}
Among the various reasons for chronic posttraumatic ulnar-sided wrist pain, instability of the distal radioulnar joint (DRUJ) has recently received major attention and finally achieved fundamental progress in understanding thanks to anatomic and biomechanical studies. This has resulted in more physiological and successful treatment methods compared with the historic options. One and the most dramatic surgical technique consists in the replacement of the main ligamentous stabilizer of the DRUJ, the so-called triangular fibrocartilage, with a tendon graft. On the basis of our experience, the refined surgical technique is presented as well as the results obtained in a retrospective consecutive case series of 48 patients with 48 wrists followed-up for 16 months in average ( 6 to 43 ) clinically and radiographically. Out of 48 unstable DRUJ's stability was restored in 44 patients associated with a significant pain relief by 1.44 points (on a scale 0 to 4 ). There was however a loss of forearm rotation of 20 degrees in average (pronation -8 degrees; supination-12 degrees), whereas the range of motion of the wrist and grip strength remained unchanged. We did not find any influence on the result by performing simultaneous ulnar shortening osteotomy, the duration of cast immobilization or the positioning in the cast. There were no postoperative complications, 4 failed cases needed secondary procedures $(2 \times$ rereconstruction, $2 \times$ Sauvé-Kapandji-operation).
\end{abstract}

Key Words: distal radioulnar joint, instability, ligament reconstruction, ulnar shortening osteotomy, ulnocarpal impaction

(Tech Hand Surg 2017;21: 107-113)

$\mathrm{M}$ any patients present with persistent ulnar-sided wrist pain following a wrist injury. If located in the distal radioulnar joint (DRUJ), this generally can be attributed to ulnocarpal impaction, distal radioulnar joint instability, distal radioulnar joint incongruity, or the combination of these.

The diagnosis of these conditions is made clinically and confirmed radiologically.

Distal radioulnar joint instability commonly, and also in this article, is understood as pathologic hypermobility between the ulna and the radius, oriented in the sagittal plane with respect to the radius. However, instability can also occur in other planes: axial (Essex-Lopresti) and frontal (radioulnar conversion). In the sagittal instability of the DRUJ, the head of the ulna dislocates volarly or dorsally out off its seat in the sigmoid notch spontaneously, upon motion or load, causing mechanical symptoms, pain, and weakness. As the bony

From the Department of Orthopedics, Division of Hand Surgery, University of Zürich, Balgrist, Zürich, Switzerland.

Conflicts of Interest and Source of Funding: The authors report no conflicts of interest and no source of funding.

Address correspondence and reprint requests to Ladislav Nagy, MD,

Department of Orthopedics, Division of Hand Surgery, University of

Zürich, Balgrist, 8008 Zürich, Forchstrasse 340, Switzerland.

E-mail: ladislav.nagy@balgrist.ch.

Copyright (C) 2017 Wolters Kluwer Health, Inc. All rights reserved. constraint contributes $<30 \%$ to the joint stability, ${ }^{1}$ relevant instability can only occur, if the ligamentous and/or the muscular stabilizers fail. The latter evidently have not been defined so far.

Because of ongoing extensive anatomic and biomechanical investigation function and dysfunction of the DRUJ including (in)stability is better and better understood.

There is unanimous evidence that the main stabilizer of the DRUJ is the triangular fibrocartilage complex (TFCC), namely the palmar and dorsal distal radioulnar ligaments. ${ }^{2-6}$ Disruption of the structural integrity of these ligaments leads to DRUJ instability, resulting in ulnar wrist pain, weakness, and mechanical symptoms. For acute TFCC tears, if not responding to conservative management, surgical repair arthroscopically or open has been recommended. If however, the TFCC presents with an irreparable rupture or the time elapsed as the injury has irreversibly weakened, shrunk, or distorted the residual TFC-tissue a simple suture is not yielding the desired result, thus a formal reconstruction is indicated.

In the past, instability of the DRUJ has been an unsolved problem and its treatment a never-ending debate. Thus, various operative techniques for restoring stability of the DRUJ have been described. ${ }^{7-17}$ The historic methods did not respect the functional anatomy and biomechanics, and logically, were not successful, but only raised the incentive for further modifications, ultimately resulting in a myriad of ultimately inappropriate procedures. The first anatomic and successful partial TFCC reconstruction has been described by Scheker et al. ${ }^{18}$ These investigators have reconstructed either the dorsal radioulnar ligaments (DRUL) or the palmar radioulnar ligaments (PRUL) with a free tendon graft according their understanding of the direction of instability and biomechanics. As the direction of instability may be difficult to determine and the structure to prevent either palmar or dorsal instability still in debate, Adams et $\mathrm{al}^{7}$ have reconstructed the PRUL and DRUL with a palmaris longus tendon graft passed through bone tunnels in the radius an ulna exiting at the anatomic insertion sites of the TFC. In their study out of 14 patients with posttraumatic DRUJ instability stability was restored in 12 and symptoms relieved. An arthroscopically assisted variant of the same procedure was published by Tse et al ${ }^{19}$ resulting in even smaller (but multiple) incisions, and a shorter period of immobilization.

Our method of an anatomic TFCC reconstruction differs only for the fixation of the tendon graft ends from the Adams technique, not for the shape and mechanism of the stabilization. It may allow higher tension on the tendon graft and more resistant fixation, thus possibly allowing less strict and shorter immobilization. We also renounced the radioulnar pintransfixation evidently without compromising the final stability result.

The purpose of this retrospective study was to evaluate the clinical outcome of an anatomic TFC reconstruction similar to Adams et $\mathrm{al}^{7}$; however, on a larger patient population 


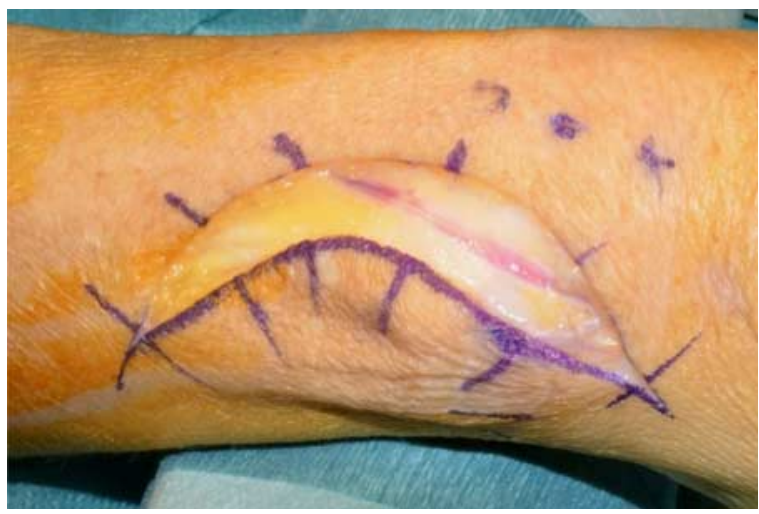

FIGURE 1. Dorsal curved incision around ulnar head. $\frac{\text { full color }}{\ln l i n e}$

and also considering different operative variants and management aspects. Therefore, we have also assessed the outcome of patients with concomitant ulnocarpal impaction, which, besides an anatomic ligament reconstruction underwent simultaneous ulnar shortening osteotomy. With respect to the postoperative management, different durations of immobilization and positions were evaluated as well as the effect of dynamic splinting.

\section{ANATOMY}

The stability of the DRUJ is provided on one side by the bony geometrical containment between sigmoid notch of distal radius and the head of the ulna head and, more importantly, by the surrounding soft tissue, including the joint capsule, the pronator quadratus, the interosseous membrane, the ECU tendon sheath and most importantly, the TFCC. ${ }^{2-4,18,20-22}$ Of the latter, the PRUL and DRUL are the main stabilizers of the DRUJ. ${ }^{2-4}$ They are also the only structures attached to the fovea of the ulnar head through which the axis of forearm rotation passes. ${ }^{19,23}$

\section{INDICATIONS}

As in our clinic the procedure was introduced in 2004, the review encloses patients treated operatively between 2004 and 2011. In this period, 220 patients with TFCC lesions were treated operatively. There were 24 isolated ulna shortenings, 41 arthroscopic or open debridements, 80 TFC repairs and finally 75 reconstructions.

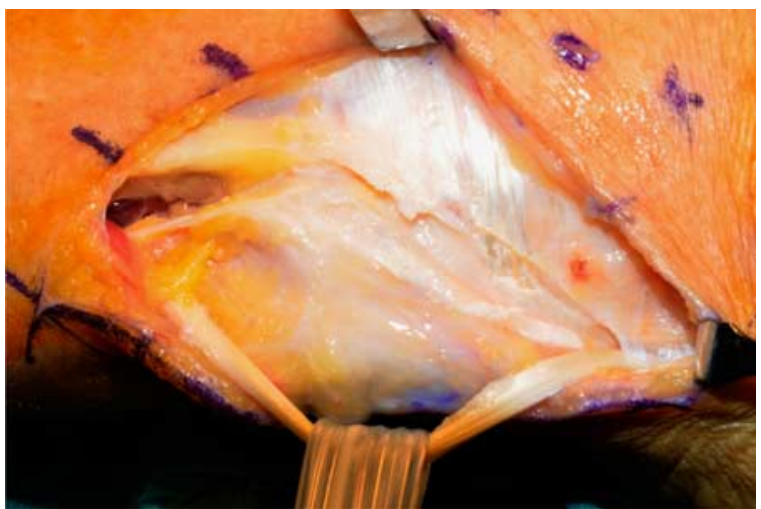

FIGURE 2. Open the retinaculum through the fifth compartment and hold away the EDQ tendon. full color

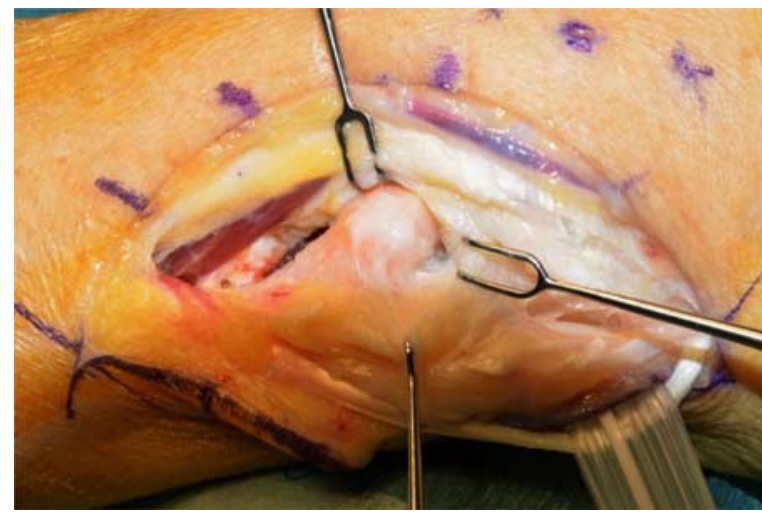

FIGURE 3. Curved arthrotomy of the distal radioulnar joint proximal to the TFC. full color

From these 75 patients with TFC reconstruction 27 patients were excluded from the study. Exclusion criteria were follow-up $<6$ months, concurrently performed radial osteotomy, radial head prosthesis, major reconstructive wrist surgery (carpal ligament repair or interposition arthroplasty).

Thus, this retrospective review is on the basis of 48 wrists in 48 patients who were treated with an anatomic reconstruction of the TFC between 2004 and 2011 performed by 2 surgeons (S.A. and N.L.). There were 27 females and 21 males with a mean age of 33.48 years (range, 14 to $62 \mathrm{y}$ ). The followup period ranged from 6 to 43 months with a mean of 15.9 months. Twenty-seven patients had ligament reconstruction alone, 21 patients with painful ulnocarpal impaction, positive ulnocarpal impaction test, positive or neutral ulnar variance underwent concurrently an ulnar shortening osteotomy.

All patients had chronic $(>6 \mathrm{mo})$ ulnar-sided wrist pain resisting conservative treatment. All patients displayed painful sagittal instability of the DRUJ, clearly increased in comparison with the opposite healthy side. Further 21 of these presented also with a painful ulnocarpal impaction. All patients had standard radiographs. Patients with evidence of degenerative arthritis of the DRUJ were excluded from the procedure.

The charts were reviewed for preoperative and postoperative wrist range of motion, grip strength, pain, and clinical stability at 6 weeks, 3 months, 6 months, and at the last follow-up.

Pain was judged by the patient on a scale from 0 to 4 $(0=$ no pain, $1=$ occasional/slight pain upon heavy loading,

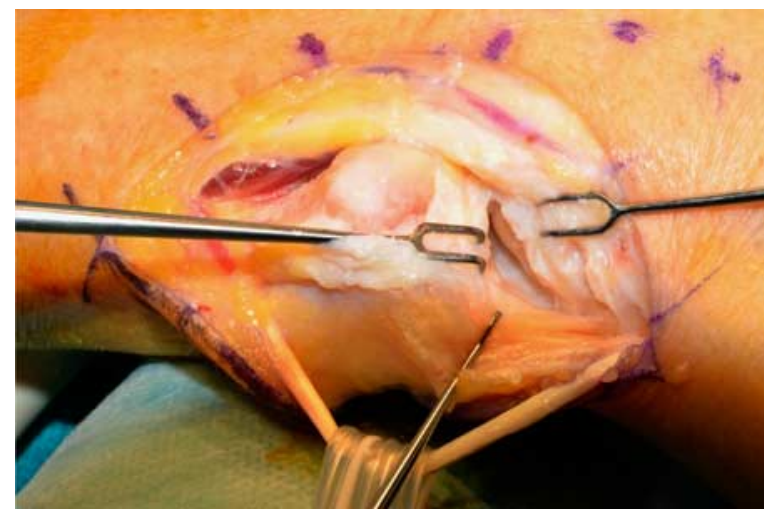

FIGURE 4. Transverse arthrotomy distal to the triangular fibrocartilage. full color

Copyright (C) 2017 Wolters Kluwer Health, Inc. All rights reserved. 


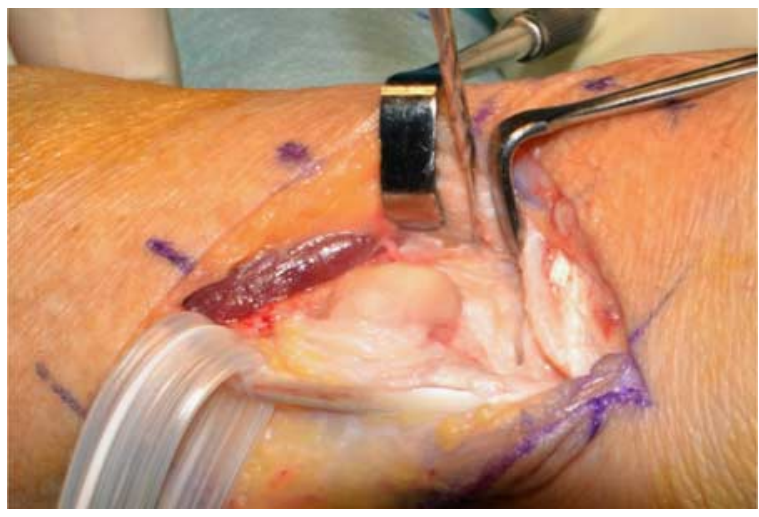

FIGURE 5. Drill hole into the radius over a guide wire. $\frac{\text { full color }}{0 \mathrm{nline}}$

$2=$ moderate pain, but only upon loading, 3 = pain evoked by activities of daily living, and $4=$ pain at rest). DRUJ stability was graded as stable, subluxing, or completely instable.

The objective clinical parameters included the DRUJ stability, expressed as anteroposterior passive translation of the DRUJ compared with the opposite wrist, range of motion of the forearm and wrist (measured with a handheld goniometer), and grip strength (Jamar, position 2). The postoperative rehabilitation and complications (intraoperative, wound healing, or infection) were reviewed from the charts.

For the statistic analysis we used a $t$ test (paired samples correlation), Pearson $\chi^{2}$ test, or Spearman rank test, whereas $P<0.05$ as significant was determined.

\section{TECHNIQUE}

The incision is dorsal, curving radially around the ulnar head, about $7 \mathrm{~cm}$ long (Fig. 1). The extensor retinaculum is opened through the fifth extensor compartment, the EDQ-tendon is mobilized and held away (Fig. 2). An arthrotomy along the dorsal lip of the sigmoid notch curving ulnarly proximal to the TFC (not injuring its fibers/the DRUL nor the sixth extensor compartment) is created (Fig. 3). This will allow for an excellent exposure of the articular surfaces of the DRUJ and the proximal surface of the TFCC especially its foveal insertion. A second, transverse arthrotomy is made just distal to the DRUL (Fig. 4). Apart the exposure of the luno-triquetral ligament (which can also be involved) the integrity of the TFCC and its potential for repair is assessed. If the TFCC is acutely ruptured off its ulnar insertion and its substance and

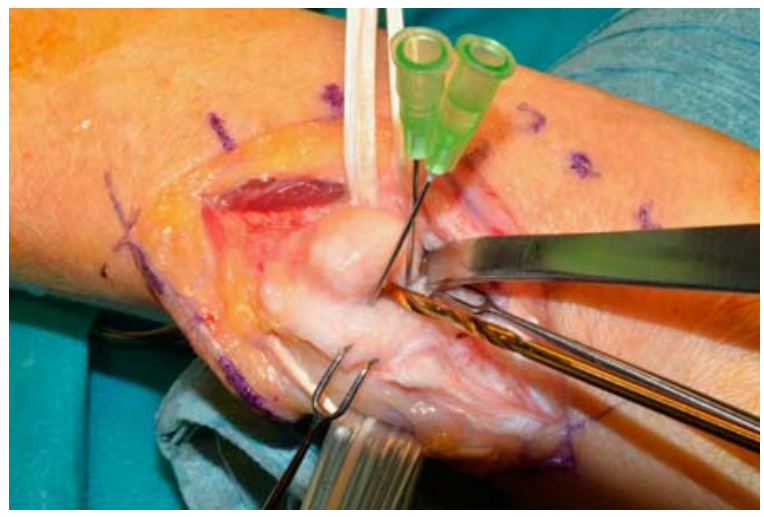

FIGURE 6. Drilling the prestyloid bone insertion, after locating the ulnar styloid. $\frac{\text { full color }}{0 \operatorname{nline}}$

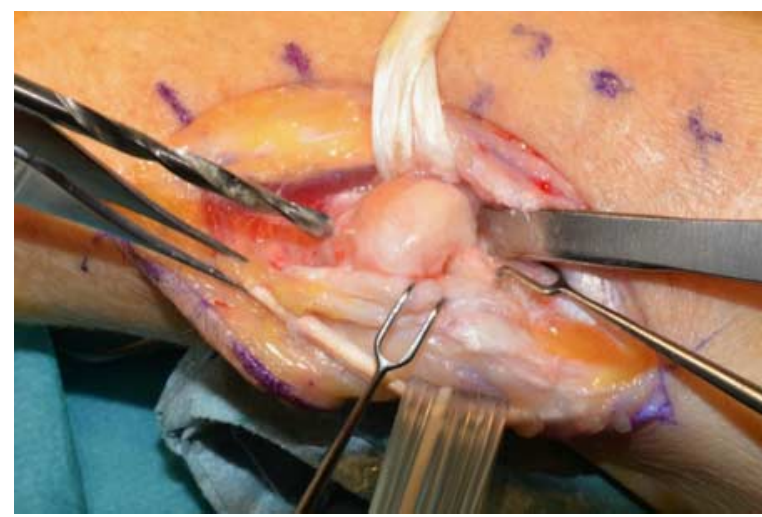

FIGURE 7. Drilling the first exit hole on ulnar neck. full color

tissue quality is not relevantly altered, a simple and direct reattachment to fovea through transosseous drill holes or with a suture anchor is performed. This should result in reestablishing the stability of the DRUJ upon intraoperative testing. If this is not the case, if the freshened TFC undersurface does not intimately reach the debrided prestyloid area of the ulnar head or its tissue quality (only scar and synovium) is insufficient to provide a stable DRUJ, a formal reconstruction of the radioulnar ligaments is necessary.

If, because of positive clinical ulnocarpal impaction test and an ulna plus variant, a concurrent corrective osteotomy of the ulna is planned, this is best done before the ligament reconstruction. This can sometimes improve DRUJ stability, thus obviating the necessity for a TFCC reconstruction.

Debridement of the scar tissue as well as freshening of the distal undersurface of the TFCC and the fovea is carried out. A second, transverse skin incision is placed ulnar at the level of the wrist flexion crease, 3 to $4 \mathrm{~cm}$ in length. Through this, the palmaris longus tendon can be harvested with a tendon stripper. Alternatively, a strip of the FCU or a long toe extensor tendon can be used.

According to the size of the graft, usually 2.5 to $3.5 \mathrm{~mm}$ in diameter, bone tunnels, one through the radius, and another in the ulna are created.

The tunnel through the radius is oriented sagittally and is located at the most distal at the most ulnar-sided corner of the radius, obviously not entering either joint. This best done with canullated drills after a guide wire has been placed and confirmed for correctness fluoroscopically (Fig. 5). The bone tunnel in the ulna runs in the long axis of the bone, with

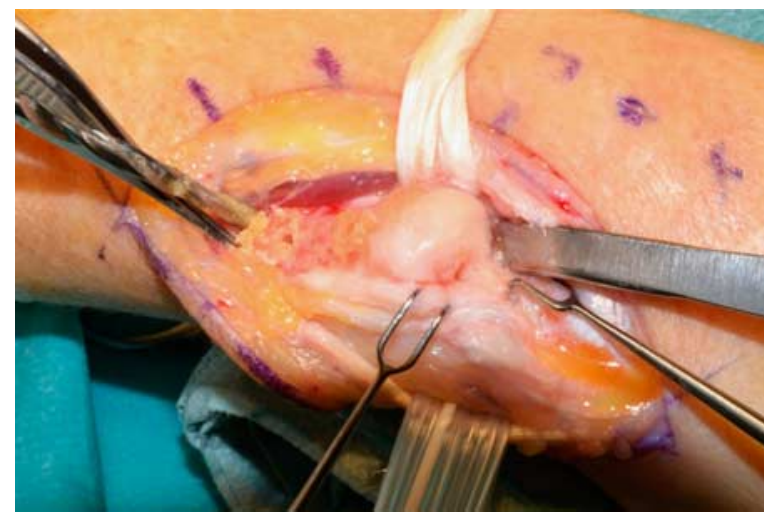

FIGURE 8. Drilling the second exit hole on ulnar neck. full color 


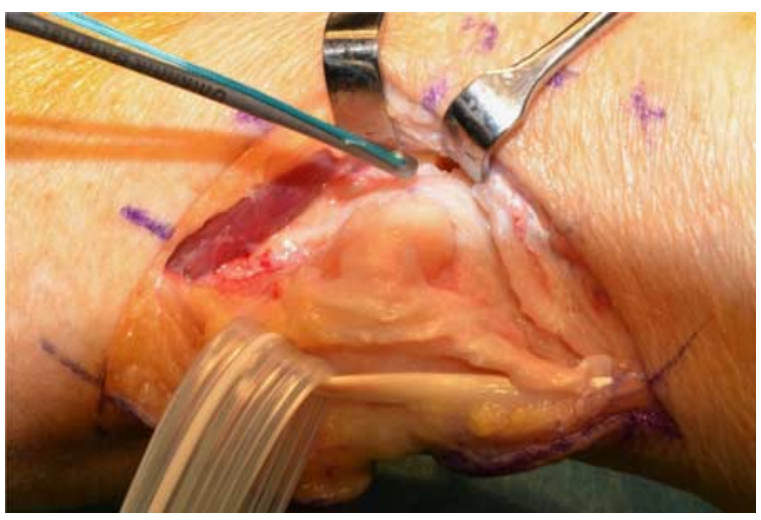

FIGURE 9. Prepare for pulling through the palmaris longus graft, insertion. $\frac{\text { full color }}{0, \operatorname{line}}$

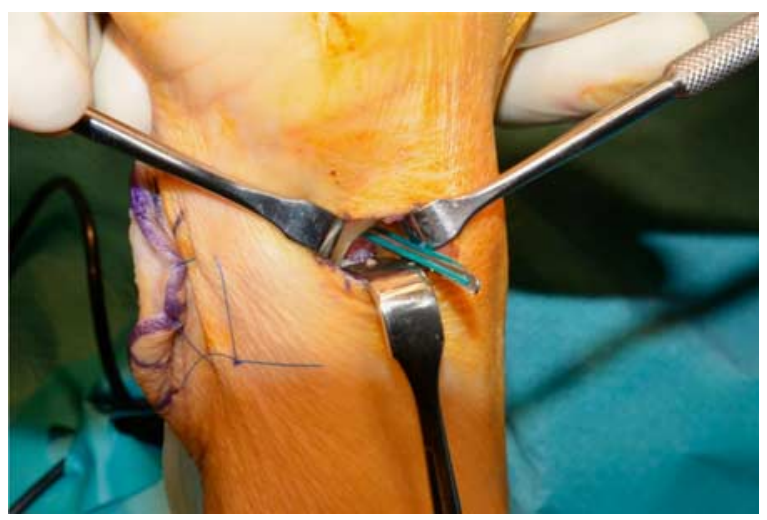

FIGURE 10. Prepare for pulling through the palmaris longus graft, exit. $\frac{\text { full color }}{\operatorname{lnl} \text { line }}$

paramount attention to the entry point, which is located in the fovea, just radial to the base of the ulnar styloid (Fig. 6). We recommend to palpate the margins of the ulnar styloid before drilling, as with the most frequent (supine) positioning of the patient with the arm abducted and the wrist pronated, the styloid is located dorsal (and not lateral/ulnar). Usually we start with a $2 \mathrm{~mm}$ drill and enlarge it to 3.2 , as it will have to accommodate 2 strands of tendon graft. The axial drill hole enters the medullar cavity of the ulna, which is reached, in addition by 2 subcapital drill holes (Figs. 7, 8). The tendon graft is pulled through the radius from palmar to dorsal

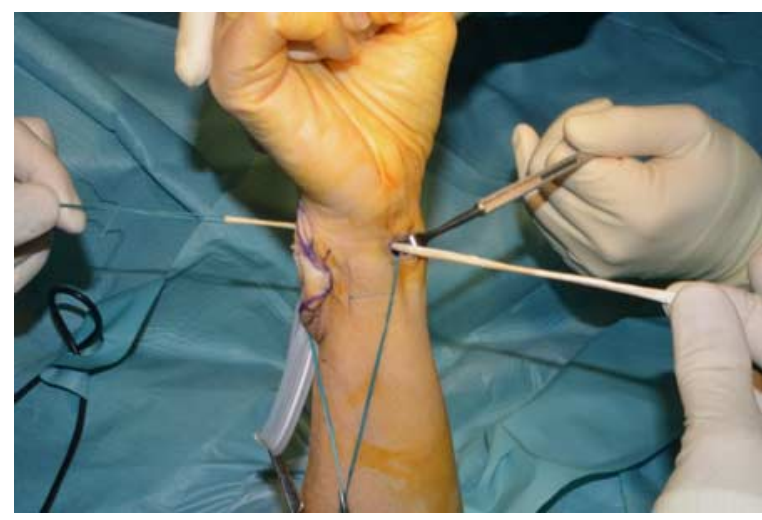

FIGURE 11. Pulling through the palmaris longus graft. $\frac{\text { full color }}{0 \ln l i n e}$

(Figs. 9-12). The palmar tail of the graft is pulled into the DRUJ through a tiny palmar arthrotomy just proximal to the TFC origin (Fig. 13). Thus, both ends of the tendon graft become visible in the DRUJ/under the TFCC remnants (Figs. 14, 15). These both ends, together with a 2-0 PDS-suture attached to the most ulnar end of the TFC are pulled into the prestyloid bone tunnel and pulled out separately through the 2 subcapital drill holes (Figs. 16, 17). In neutral forearm rotation, the tendon ends and the sutures now are maximally tensioned, tied, and secured over the bone bridge between the 2 drill holes (Fig. 18). This results in firm stability of the DRUJ and free prosupination. Finally, the 2 arthrotomies are closed with separate resorbable running sutures, the EDQ is relocated and the extensor retinaculum closed at a tension ensuring that the ECU is reduced in its groove. After wound closure, a long arm cast is applied in neutral or supinated forearm position.

The long arm cast is routinely maintained for 6 weeks in total. During the initial 4 the wrist and forearm are completely immobilized, then cautious pronation and supination exercises are begun removing the cast 3 to 4 times for some 15 minutes every day. From the seventh week, the cast is only used for exposed activities otherwise left away and light protected use of the hand is allowed, active-assistive motion exercises are continued and, if necessary, a short cast or dynamic splint is used. Strengthening exercises are not allowed before 12 weeks, then also, according the consolidation of the concurrent ulna osteotomy, full weight bearing is permitted.

In our cohort, postoperative immobilization was in neutral rotation in 42 patients and moderately (30 to 40 degrees) supinated in 6 patients for an average duration of 4.73 weeks (range, 2 to $6 \mathrm{wk}$ ). Wrist mobilization was started at an average of 4.73 weeks (range, 2 to $6 \mathrm{wk}$ ). Depending upon the progress
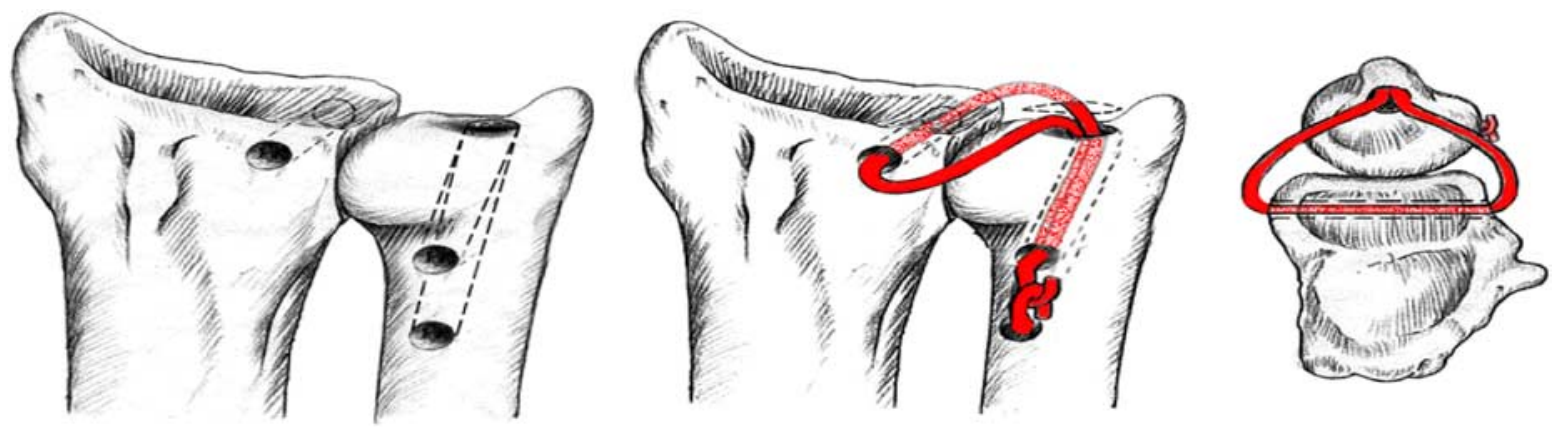

FIGURE 12. Drill holes. $\frac{\text { full color }}{0 n \operatorname{line}}$ 


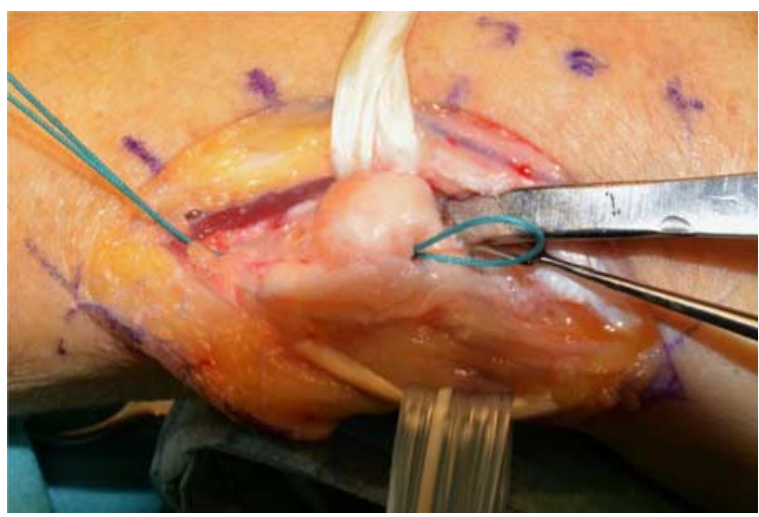

FIGURE 13. Prepare for pulling through the palmaris longus graft. $\frac{\text { full color }}{\operatorname{lnline} \mid}$

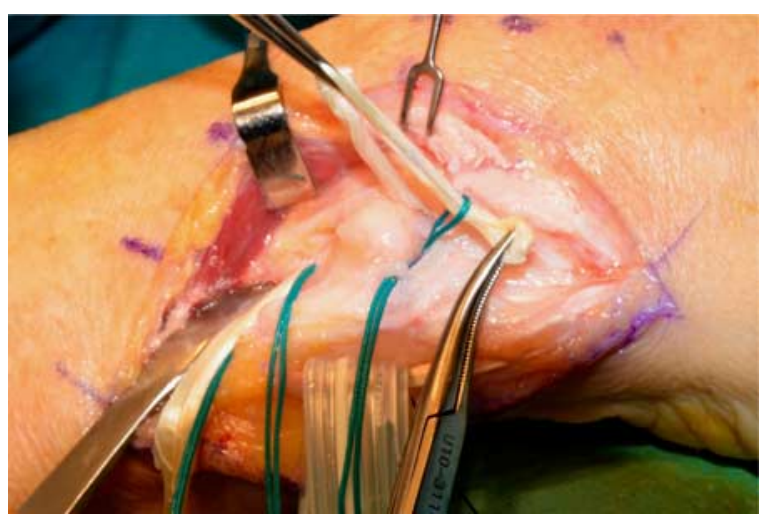

FIGURE 14. Prepare for pulling through the first slip of the palmaris longus graft. full color

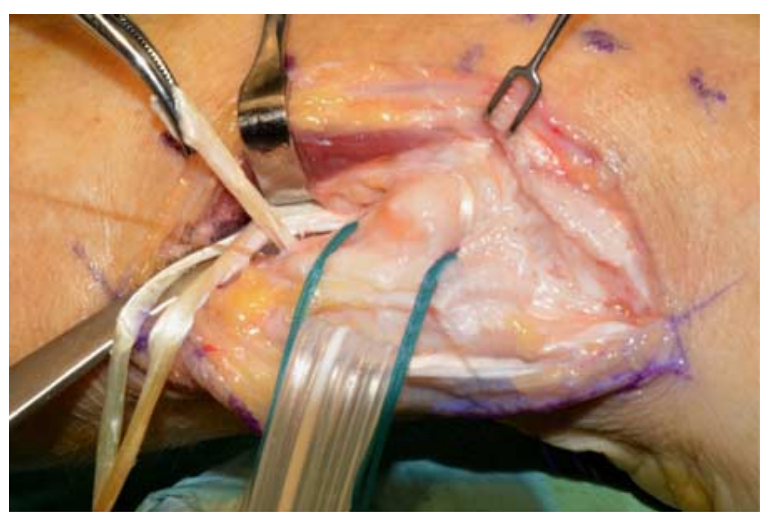

FIGURE 15. First first slip of the palmaris longus graft pulled through, exiting at the proximal exit hole on ulnar neck. full color

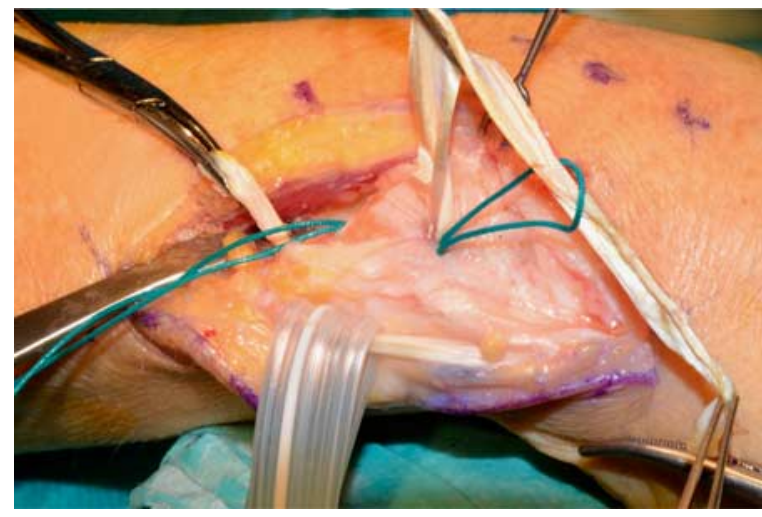

FIGURE 16. Prepare for pulling through the second slip of the palmaris longus graft. $\frac{\text { full color }}{0,\lfloor i n e}$

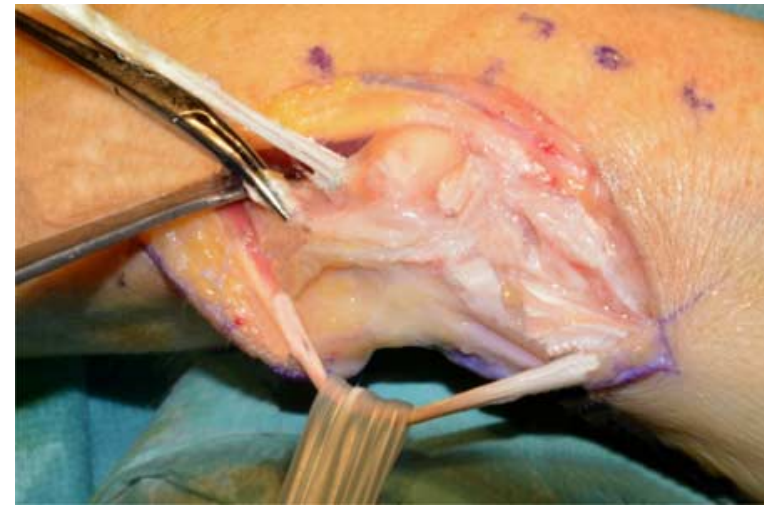

FIGURE 17. Second first slip of the palmaris longus graft pulled through, exiting at the distal exit hole on ulnar neck. $\frac{\text { full color }}{\ln l i n \mathrm{e}}$

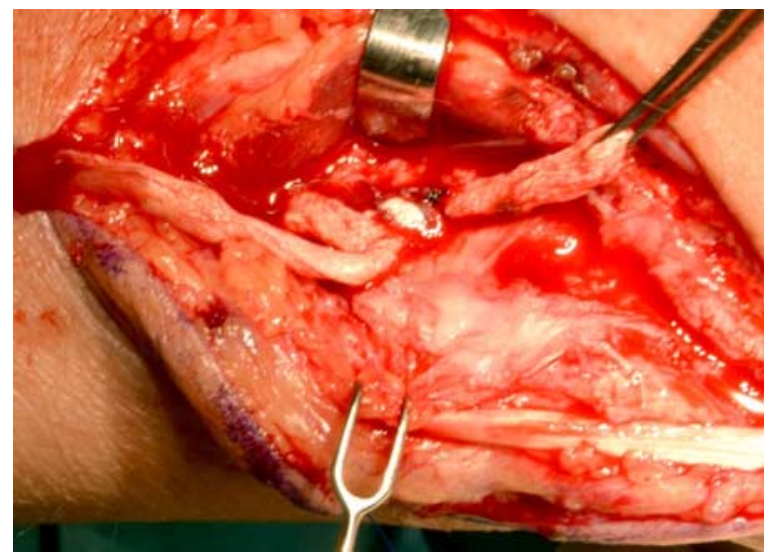

FIGURE 18. The 2 slips of the palmaris longus graft tightened and knotted. full color 


\begin{tabular}{|c|c|c|c|c|c|c|c|c|}
\hline Mean & Preoperative & $6 \mathrm{mo}$ & $\triangle$ & $12 \mathrm{mo}$ & $\triangle$ & Last & $\triangle$ & Preoperative to Last (\%) \\
\hline Pronation (deg.) & 76.4 & $69.1 *$ & $-7.3^{*}$ & $66.9 *$ & $-9.5^{*}$ & $67.9 *$ & $-8.5^{*}$ & 88.9 \\
\hline Supination (deg.) & 78.5 & $63.7^{*}$ & $-14.9 *$ & $66.0 *$ & $-12.5^{*}$ & $66.1 *$ & $-12.5^{*}$ & 84.1 \\
\hline Flexion (deg.) & 70.2 & 66.3 & -4.0 & 68.5 & -1.7 & 67.1 & -3.1 & 95.5 \\
\hline Extension (deg.) & 71.5 & 67.5 & -4.0 & 70.1 & -1.4 & 70.1 & -1.1 & 98.0 \\
\hline Radialduction (deg.) & 18.8 & 21.8 & 3.1 & 22.0 & 3.2 & 19.6 & 0.9 & 104.5 \\
\hline Ulnarduction (deg.) & 35.7 & 34.0 & -1.7 & 32.8 & -3.0 & 32.9 & -2.8 & 92.2 \\
\hline Grip strength (contralateral) $(\mathrm{kg})$ & $24.6 / 38.5$ & $28.8 / 40.0$ & 4.2 & $32.1 / 40.3$ & 7.4 & $31.0 / 38.6$ & 6.4 & $125.8 / 100.5$ \\
\hline Pain & 2.6 & 1.7 & & 1.2 & & 1.3 & & \\
\hline Stability & 1.4 & & & & & 0.1 & & \\
\hline
\end{tabular}

*Significance level $P>0.05$ reached.

of mobility, a dynamic splint (destined to improve supination or pronation) was installed in 37 patients at average of 6.65 weeks postoperatively (range, 5 to $12 \mathrm{wk}$ ).

Postoperatively, 8 patients had removal of the plate used for the ulna shortening osteotomy. Two patients with failed repair required a Sauvé-Kapandji procedure, in other 2 patients a repeat TFCC reconstruction was necessary. Two patients claimed paresthesia in the distribution of the ulnar nerve, thus 1 patient had an exploration and neurolysis of the ulnar nerve. None of the patients developed a postoperative infection or regional pain syndrome.

\section{EXPECTED OUTCOMES}

The mean follow-up period was 15.9 months (6 to 43 mo).

The average pain score was reduced from 2.6 (preoperatively) to 1.7 (6 mo postoperative) and 1.2 (12 mo postoperative) (Table 1).

By objective testing, DRUJ stability was restored in 44 patients, whereas in 2 patients a subluxation and in further 2 patients a frank persistent instability was noted. At all postoperative follow-up visits the range of motion showed a significant reduction of pronation and supination, also after 6 months, 12 months, and at the latest follow-up. Average pronation range dropped from 76.4 degrees (preoperative) to 69.1 degrees (6 mo), 66.9 degrees (12 mo), and 67.9 degrees (latest follow-up). Supination decreased from 78.5 degrees (preoperative) to 63.7 degrees (6 mo), 66.0 degrees (12 mo), and 66.1 degrees (latest follow-up) (Table 1).

There was no significant change in the range of motion for flexion, extension, and radial deviation (Table 1). Only for ulnar deviation there was a marginal loss compared with preoperative (35.7 to 32.9 degrees) and this only in the latest follow-up.

The concurrent ulnar shortening osteotomy had no significant impact on the preoperative and postoperative pain and instability. In contrast, its relationship with the range of motion was significant: there was an increased loss of supination and flexion at 6 months; however, interestingly, the osteotomy patients have had a significantly better preoperative supination ( 88.8 vs. 74.5 degrees; $P=0.0005$ ); thus, in spite of the greater loss it also had a significantly better final supination (72.9 vs. 65.3 degrees; $P=0.04)$. The average supination range with ulnar shortening osteotomy dropped from 88.8 degrees (preoperative) to 63.3 degrees $(6 \mathrm{mo})$, whereas the average flexion range with ulnar shortening osteotomy dropped from 73.0 degrees (preoperative) to 62.5 degrees $(6 \mathrm{mo})$.

The postoperative positioning of the forearm in the cast (in neutral or supination) position as well as the use of postoperative dynamic splints had no significant effect on the postoperative range of motion, pain, or stability in the DRUJ. Patients with significant worse (26.3 vs. 41.4 degrees; $P=0.0003)$ and loss (56.7 vs. 40.5 degrees; $P=0.006)$ of supination at 6 weeks received a dynamic splint. The resulting improvement (41.1 vs. 33.6 degrees) was not significant $(P=0.08)$. Thus these patients, in spite of the splinting ended up with less supination, (67.3 vs. 75.0 degrees; $P=0.03$ ) respectively had more loss of supination (15.6 vs. 6.8 degrees; $P=0.02)$.

The amount of postoperative wrist extension showed a significant dependence upon the duration of immobilization. There was a significant increase in wrist extension from preoperative compared with postoperative (both 6 mo and latest follow-up) with a longer period of immobilization in a long arm cast. With $>4$ weeks of immobilization average wrist extension increased from 61.1 degrees (preoperative) to 67.5 degrees (12 mo) and 77.6 degrees (latest follow-up); whereas with $<4$ weeks of immobilization it decreased from 73.9 degrees (preoperative) to 70.3 degrees $(12 \mathrm{mo})$ and 70.0 degrees (latest follow-up), which was statistically significant $(P=0.043$ and 0.029 , respectively)

Comparing our results with those of Adams, we have almost the same amount of postoperative loss in the forearm rotation ( 20 vs. 23 degrees), divided in reduction of pronation was 8.5 degrees (Adams, 10 degrees) respectively supination 12.5 degrees (Adams, 13 degrees).

\section{COMPLICATIONS}

Postoperatively a subluxation persisted in 2 patients, whereas in 2 further patients a frank persistent instability was noted. This 4 failed cases needed secondary procedures $(2 \times$ rereconstruction, $2 \times$ Sauvé-Kapandji-operation).

No other complications (eg, nerve injuries or nonunion) were seen with this technique.

\section{REFERENCES}

1. Stuart PR, Berger RA, Linscheid RL, et al. The dorsopalmar stability of the distal radioulnar joint. J Hand Surg Am. 2000;25:689-699.

2. Kihara H, Short WH, Werner FW, et al. The stabilizing mechanism of the distal radioulnar joint during pronation and supination. J Hand Surg Am. 1995;20:930-936.

3. Palmer AK, Werner FW. The triangular fibrocartilage complex of the wrist - anatomy and function. J Hand Surg Am. 1981;6:153-162.

4. af Ekenstam F, Hagert CG. Anatomical studies of the geometry and stability of the distal radioulnar joint. Scand J Plast Reconstr Surg. 1985;19:17-25. 
5. Nicolaidis SC, Hildreth DH, Lichtman DM. Acute injuries of the distal radioulnar joint. Hand Clin. 2000;16:449-459.

6. Tolat AR, Stanley JK, Trail IA. A cadaveric study of the anatomy and stability of the distal radioulnar joint in the coronal and transverse planes. J Hand Surg Br. 1996;21:587-594.

7. Adams BD, Berger RA. An anatomic reconstruction of the distal radioulnar ligaments for posttraumatic distal radioulnar joint instability. J Hand Surg Am. 2002;27:243-251.

8. Shih JT, Lee HM. Functional results post-triangular fibrocartilage complex reconstruction with extensor carpi ulnaris with or without ulnar shortening in chronic distal radioulnar joint instability. Hand Surg. 2005;10:169-176.

9. Teoh LC, Yam AK. Anatomic reconstruction of the distal radioulnar ligaments: long-term results. J Hand Surg Br. 2005;30:185-193.

10. Gemmill JF. On the movement of the lower end of the radius in pronation and supination, and on the interosseus Membrane. $J$ Anat Physiol. 1900;35:101-109.

11. Schuind F, An KN, Berglund L, et al. The distal radioulnar ligaments: a biomechanical study. J Hand Surg Am. 1991;16:1106-1114.

12. Adams BD. Partial excision of the triangular fibrocartilage complex articular disk: a biomechanical study. J Hand Surg. 1993;18A:334-340.

13. King GJ, McMurtry RY, Rubenstein JD, et al. Kinematics of the distal radioulnar joint. J Hand Surg. 1986;11A:711-717.

14. Bowers WH. The distal radioulnar joint. In: Hotchkiss RN, Peterson WC, ed. Green's Operative Hand Surgery. Philadelphia: Churchill Livingstone; 1999:986-1014.
15. Breen TF, Jupiter JB. Extensor carpi ulnaris and flexor carpi ulnaris tenodesis of the unstable distal ulna. $J$ Hand Surg Am. 1989;14:612-617.

16. Fulkerson JP, Watson HK. Congenital anterior subluxation of the distal ulna. A case report. Clin Orthop Relat Res. 1978;131: 179-182.

17. Hermansdorfer JD, Kleinman WB. Management of chronic peripheral tears of the triangular fibrocartilage complex. J Hand Surg Am. 1991;16:340-346.

18. Scheker LR, Belliappa PP, Acosta R, et al. Reconstruction of the dorsal ligament of the triangular fibrocartilage complex. $J$ Hand Surg Br. 1994;19:310-318.

19. Tse WL, Lau SW, Wong WY, et al. Arthroscopic reconstruction of the triangular fibrocartilage complex (TFCC) with tendon graft for chronic DRUJ instability. Injury. 2013;44:386-390.

20. Hui FC, Linscheid RL. Ulnotriquetral augmentation tenodesis: a reconstructive procedure for dorsal subluxation of the distal radioulnar joint. J Hand Surg Am. 1982;7:230-236.

21. Petersen MS, Adams BD. Biomechanical evaluation of distal radioulnar reconstructions. J Hand Surg Am. 1993;18: $328-334$.

22. Johnston Jones K, Sanders WE. Posttraumatic radioulnar instability: treatment by anatomic reconstruction of the volar and dorsal radioulnar ligaments. Orthop Trans. 1995-1996;19:832.

23. Tsai TM, Stilwell JH. Repair of chronic subluxation of the distal radioulnar joint (ulnar dorsal) using flexor carpi ulnaris tendon. $J$ Hand Surg Br. 1984;9:289-294. 